\title{
Autonomous Navigation Applying Dynamic-Fuzzy Cognitive Maps and Fuzzy Logic
}

\author{
Márcio Mendonça ${ }^{1}$, Ivan Rossato Chrun ${ }^{1}$, Lúcia Valéria Ramos de Arruda ${ }^{2}$, \\ and Elpiniki I. Papageorgiou ${ }^{3}$ \\ ${ }^{1}$ Departamento de Engenharia Elétrica, Universidade Tecnológica Federal do Paraná \\ Av. Alberto Carrazai 1640, CEP 86300-000, Cornelio Procópi, Paraná, Brasil \\ mendonca@utfpr.edu.br, ivanchrun@gmail.com \\ ${ }^{2}$ Laboratório de Automação e Sistema de Controle Avançado (LASCA), \\ Universidade Tecnológica Federal do Paraná \\ Av. Sete de Setembro 3165, CEP 80230-901, Curitiba, Paraná, Brasil \\ lvarruda@utfpr.edu \\ ${ }^{3}$ Technological Education Institute of Lamia, \\ Department of Informatics and Computer Tech-nology, Lamia, Greece \\ epapageorgiouateilam.gr
}

\begin{abstract}
This work develops a knowledge based system to autonomous navigation using Fuzzy Cognitive Maps (FCM). A new variant of FCM, named Dynamic-Fuzzy Cognitive Maps (D-FCM), is used to model decision tasks and/or make inference in the robot or mobile navigation. Fuzzy Cognitive Maps is a tool that model qualitative structured knowledge through concepts and causal relationships. The proposed model allows representing the dynamic behavior of the mobile robot in presence of environment changes. A brief review of correlated works in the navigation area by use of FCM is presented. Some simulation results are discussed highlighting the ability of the mobile to navigate among obstacle (navigation environment). A comparative with Fuzzy Logic and future works are also addressed.
\end{abstract}

Keywords: Mobile Robot Navigation, Fuzzy Cognitive Maps, Dynamic-Fuzzy Cognitive Maps, Intelligent decision systems.

\section{Introduction}

Artificial Intelligence (AI) has applications and development in various areas of knowledge, such as mathematical biology neuroscience, computer science and others. The research area of intelligent computational systems aims to develop methods that try to mimic or approach the capabilities of humans to solve problems. These news methods are looking for emulate human's abilities to cope with very complex processes, based on inaccurate and/or approximated information. However, this information can be obtained from the expert's knowledge and/or operational data or behavior of an industrial system [1]. 
Researches in autonomous navigation are in stage of ascent. Autonomous Systems has the ability to perform complex tasks with a high degree of success [2]. In this context, the complexity involved in the task of trajectory generation is admittedly high efficient and, in many cases, requires that the autonomous system is able to learn a navigation strategy through interaction with the environment [3].

There is a growing interest in the development of autonomous robots and vehicles, mainly because of the great diversity of tasks that can be carried out by them, especially those that endanger human health and/or the environment, [4] and [5]. As an example, we can cite Mandow et al. [6], which describes an autonomous mobile robot for use in agriculture, in order to replace the human worker, through inhospitable activities as spraying with insecticides.

The problem of mobile robots control comprises two main sub problems: 1) navigation, determining of robot/vehicle position and orientation at a given time, and 2) guided tours, which refers to the control path to be followed by the robot/vehicle. In some cases have more complexity, example in [7] a Subsumption Architecture to develop dynamic cognitive network-based models it used for autonomous navigation with different goals: avoid obstacles, exploration and reach targets.

This work specifically proposes the development of an autonomous navigation system that uses heuristic knowledge about the behavior of the robot/vehicle in various situations, modeled by fuzzy cognitive maps [8]. In this case, the robot/vehicle determine a planning or generation of sequences of action in order to reach a given goal state from a predefined starting state.

Through cognitive maps, beliefs or statements regarding a limited knowledge domain are expressed through language words or phrases, interconnected by simple relationship of cause and effect (question/non-question). In the proposed model, the FCM relationships are dynamically adapted by rules that are triggered by the occurrence of special events. These events must change mobile behavior. There are various works in the literature that model heuristic knowledge necessary for decision-making in autonomous navigation, by means of Fuzzy systems [9], [10], [11], [12], [13] and [14]. In a similar way, the approach proposed in this paper is to build qualitative models to mobile navigation by means of fuzzy systems. However the knowledge is structured and built as a cognitive map that represents the behavior of the mobile.

Thus, the proposed autonomous navigation system must be able to take dynamic decisions to move through the environment and sometimes it must change the trajectory as a result of an event. For this the proposed FCM model must aggregate discrete and continuous knowledge about navigation. Actions such as the decision to turn left or right when sensors accuse obstacles and accelerate when there is a free path are always valid control actions in all circumstances. In this way, this type of action is modeled as causal relationship in a classical FCM.

However, there are specific situations, such as the need to maintain a trend of motion mainly in curves when the vehicle is turning left and sensors to accuse a new obstacle in the same direction. Due to inertia and physical restrictions, the mobile cannot abruptly change direction; this type of maneuver must be carefully executed. In this context, some specific situations should also be modeled on the map by causal relationships and concepts, but they are valid just as a result of a decision-making task 
caused by ongoing events. To implement such a strategy, new type of relationships and concepts will be added to the FCM classic model.

This new type of FCM in which the concepts and relationships are valid as a result of decision driven by events modeled by rules and is called Dynamic-FCM. Specifically, the work of Mendonça et al. [15] presents a type of D-FCM, which aggregates the occurrence of events and other facilities that makes appropriate this type of cognitive map, for the development of intelligent control and automation in an industrial environment.

The remainder of the paper is organized as following. Session 2 introduces Fuzzy Cognitive Maps concepts and provides a brief review of its application in autonomous navigation. Session 3 describes the proposed D-FCM and develops the autonomous navigation system. Session 4 presents simulation results obtained with the proposed navigation system and fuzzy logic navigation system and session 5 concludes the paper.

\section{Fuzzy Cognitive Maps}

Cognitive maps were initially proposed by Axelrod [16] to represent words, thoughts, tasks, or other items linked to a central concept and willing radially around this concept. Axelrod developed also a mathematical treatment for these maps, based in graph theory, and operations with matrices. These maps can thus be considered as a mathematical model of "belief structure" of a person or group, allowing you to infer or predicting the consequences that this organization of ideas represented in the universe.

This mathematical model was adapted for inclusion of Fuzzy logic uncertainty through by Kosko [8] generating widespread fuzzy cognitive maps. Like the original, FCMs are directional graph, in which the numeric values are fuzzy sets or variables. The "graph nodes", associated to linguistic concepts, are represented by fuzzy sets and each "node" is linked with other through connections. Each of these connections has a numerical value (weight), which represents a fuzzy variable related to the strength of the concepts.

The concepts of a cognitive map can be updated through the iteration with other concepts and with its own value. In this context, a FCM uses a structured knowledge representation through causal relationships being calculated mathematically from matrix operations, unlike much of intelligent systems whose knowledge representation is based on rules if-then type. However, due to this "rigid" knowledge representation by means of graph and matrix operation, the FCM based inference models lack robustness in presence of dynamic modifications not a priori modeled [17]. To circumvent this problem, this article develops a new type of FCM in which concepts and causal relationships are dynamically inserted into the graph from the occurrence of events. In this way, the dynamic fuzzy cognitive map model is able to dynamically acquire and use the heuristic knowledge. The proposed D-FCM and its application in autonomous navigation will be developed and validated in the following sections. 


\subsection{FCM in Intelligent Obstacle Navigation}

Some related works which use cognitive maps in the robotics research area can be found in the literature. Among them, we can cite the work in [12] that employs probabilistic FCM in the decision-making of a robot soccer team. These actions are related to the behavior of the team, such as kick the ball in presence of opponents. The probabilistic FCM aggregates a likelihood function to update the concepts of the map. A Fuzzy cognitive map is used by [14] and [13] to guide an autonomous robot. The FCM is designed from a priori knowledge of experts and afterwards it is refined by a genetic algorithm.

Despite of the use of a known trajectory, actions are necessary due to errors and uncertainties inherent in the displacement of the robot, such as slippage, reading errors of the sensors, among others. A review of other related works employing intelligent navigation in robotics can be found in [14]. This paper also presents a Cognitive Map to implement a 3-D representation of the environment where an autonomous robot must navigate. The described architecture use a previously stored neural network based model to implement adjustments and course corrections of the robot in presence of noise and sensor errors. Similar to these works, we also use a fuzzy cognitive map to navigation tasks.

However our navigation system does not use a priori information about the environment. The FCM represent the usual navigation actions as turn right, turn left, accelerate and others. The adaptation ability to environment changes and to take decisions in presence of random events is reached by means of a rule based system. These rules are triggered in accordance of "intensity" of the sensor measurements.

\section{The D-FCM Model}

The development of a FCM model follows the steps of the work [15]. In this case, we identify 3 inputs related to the description of the environment (presence of obstacles) and 3 outputs describing the mobile's movements: turn left, turn right and move forward. The three inputs take values from the three sensors located at left, right and front side of the mobile.

These concepts are connected by arcs representing the actions of acceleration (positive) and braking (negative). Three decisions are originally modeled, if left sensor accuses an obstacle in this position, the vehicle must turn to the right side and equally if the right sensor accuses an obstacle in the right side, the vehicle turns to the other side. The direction change decision implies smoothly vehicle deceleration. The third decision is related to a free obstacle environment; in this case the mobile follows a straight line accelerating smoothly.

The figure 1 show the D-FCM modeled. The input concepts are SL, SR and SF and the output concepts are OutLeft, OutRigth and OutFront. The values of the concepts are the readings of the corresponding sensors. As a fuzzy number, these values are normalized into the interval $[0,1]$. The relationships among these concepts are modeled by weights w1 to w5 which are computed. It is worthwhile to note in figure 1, that the concepts O.L. (-1) and O.R. (-1) are the values of the concepts in the previous 
state. This representation is equivalent to insert negative values (-1) in the corresponding diagonal positions of matrix $\mathrm{W}$.

We choose to retain this representation to highlight that some concepts has memory. In this case, the mobile can remember the actions taken to turn left or right to prevent a zig-zag motion. As a result the mobile can maintain a movement trend.

In order to model the adaptation ability, we introduce 3 new concepts into the FCM associated to an "intensity" of motion (acceleration or braking) at each direction. There are left factor, right factor and front factor in figure 1. The factor concepts have their values changed according to the current condition of the vehicle motion and the occurrence of events. These events are modeled by the weights ws (ws1, ws 2 and ws3) in figure 1 which are obtained by applying the rules of type IF-THEN based on linguistic terms. These rules represent some decisions such as if the mobile is turning right because the left sensor has detected an obstacle and suddenly the right sensor also detects an obstacle then the factor right is small (ws3). The default value of the factor concepts is one. If any rule is triggered the weights ws are null. Finally the outputs of the D-FCM are the product between the factor concepts and the output of classical FCM (OutLeft, OutRigth and OutFront).

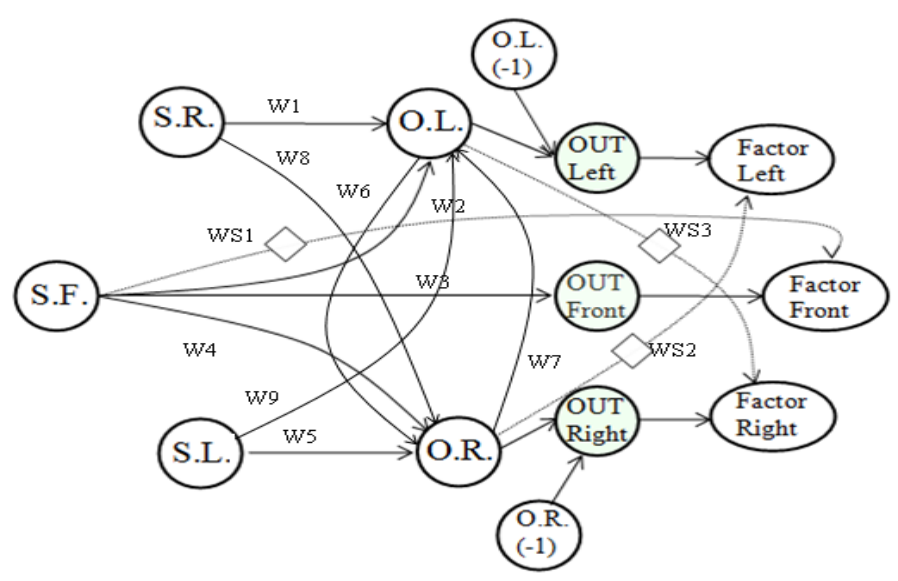

Fig. 1. The proposed D-FCM

In conclusion, the proposed D-FCM navigation system confers to the robot/vehicle the following behavior:

- The mobile is autonomous and it moves into unknown environment from an origin point to an end point.

- If an obstacle is detected by the front, left and/or right sensors the mobile must take a decision about new direction to follow.

- Default navigation position is in a straight line with constant speed, i.e. lateral movements are used only as a result of obstacle detection.

- When the mobile is in motion and the sensors don't identify any obstacle, the mobile accelerates smoothly and then it remains in a constant speed. 
- Motion trend corresponds to an average between the current movement values and the values in immediately before instant, which prevents any sharp changes in direction of the mobile navigation.

- When the mobile is turning in left or right direction and the opposite sensor detects an obstacle, the motion trend is maintained but the mobile is softly breaking until to reestablish a straight movement.

For example if the sensors don't detect any obstacle then the mobile will accelerate in a straight line. The output related to turning will have no value related to the decision. If while he is moving forward the right sensor detects an obstacle then the D-FCM will make the decision to turn left and slowly break and turn left. In this case the front factor will be reduced and the output related to turn right will have no effect. And if while he is turning left to deviate from the obstacle the left sensor detects an obstacle the mobile tends to maintain his movement but the mobile will turn less to right since the factor will take new values and the output related to turn right will now take effect in his decision making.

Intelligent control architecture to the navigation system is similar [15]. The input interface read the sensor measurements which are inversely proportional to the distance of obstacles.

In order to dynamic adapt the D-FCM weights we use the hebbian learning algorithm for FCM that is an adaptation of the classic hebbian method. Different proposals and variations of this method applied in tuning or in learning for FCM are known in the literature [18].

$$
W_{i}(k)=W_{i j}(k-1) \pm \gamma \Delta A_{i}
$$

Where: $W_{i}(k)$ is a new value of the weight, $W_{i j}(k-1)$ old value, $\gamma$ factor forget and $\Delta \mathrm{Ai}$ is a variation of the same concepts in two steps.

In this paper, the method is used to update the intensity of causal relationships in a deterministic way according to the variation or error in the intensity of the concept or input variable. In [15] one similar proposal for dynamic tuning in FCM uses Reinforcement Learning Algorithm (Q-learning). In this case, the application of Hebbian learning provides control actions as follows: if an obstacle to the right is nearest the causal relationship of exit turn left increases and consequently increases its control action. The others action have same behavior. Forgetting factors were obtained from empirical mode. And finally minimum and maximum limits were placed due to the application of the method is to tune the dynamic D-FCM, thereby varying the intensity of causal relationships should be within a clearly defined range. The range was chosen by observation of the mobile behavior in the environment. This range is defined by observation of the dynamic actions and closed intervals, $[0.35,0.65]$ for $W_{F}$, $[0.6,1]$ for $W_{E}$ and $[0.6,1]$ for $W_{D}$.

$$
\begin{gathered}
W_{F}=W_{\text {Fold }}(i)-0.7 * \operatorname{erroF}(i) \\
W_{E}=W_{\text {Eold }}(i)-0.7 * \operatorname{erroE}(i) \\
W_{D}=W_{\text {Dold }}(i)-0.7 * \operatorname{erroD}(i)
\end{gathered}
$$


Where: $W_{F}$ is the weight related to front proximity sensor, $W_{E}$ is the weight related to left proximity sensor and $W_{D}$ is the weight related to the right proximity sensor.

A system of autonomous navigation using fuzzy logic was implemented in order to assess performance, outcomes and differences in acquisition and processing of empirical knowledge used in developing the tool presented fuzzy logic. The Work of [19] is similar and presents a fuzzy control strategy similar. The Fuzzy system is implemented in this work is type Mandani with 3 inputs, 3 outputs and employs 23 rules for abstraction of the same heuristic logic navigation controller inserted in the D-FCM. The inputs are the sensors, right, left and front and outputs are turning right, left and accelerate. These rules were implemented in an intuitive way according to heuristic D-FCM. For example:

- IF the right sensor is strong then turn left strong.

- IF the right sensor is weak then turn left weak.

- IF right sensor and frontal sensor very strong then weak accelerate and turn left very strong

- IF right, left and frontal sensor weak then accelerate Strong.

The strategies of decision making are the same, the difference in this formalism. In D-FCM knowledge is structured in the form of a graph, while the Fuzzy model is based on classical Fuzzy rules.

\section{Characterization/Simulation Results}

A 2-d animation simulated environment has inspired in real case (figure 2) and designed to test and validate our proposed navigation system.

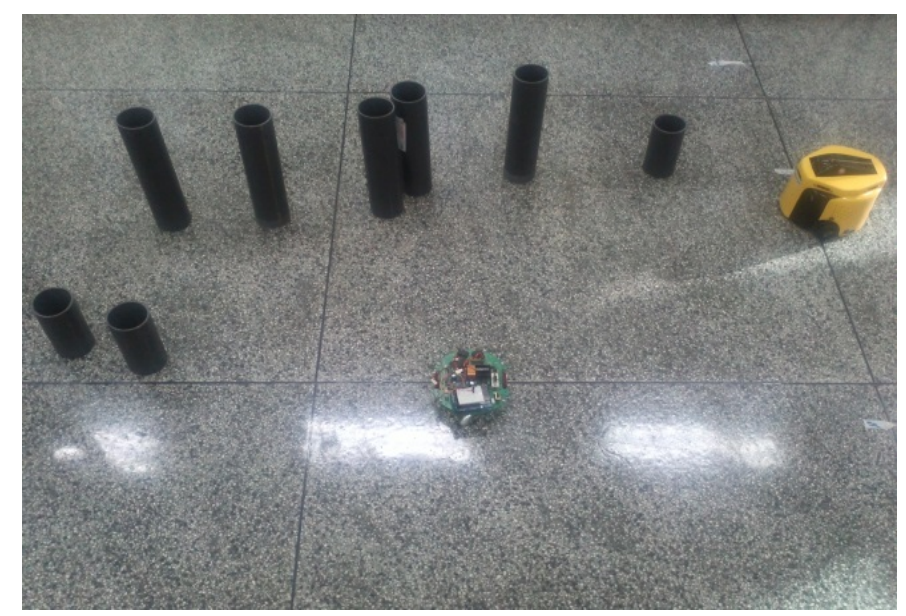

Fig. 2. Real Environment with Dynamic Obstacle 
The kinematic equations simulating the robot dynamic behavior has been inspired by [10] and uses scale in centimeters. In fact, the simulated robot corresponds to a mobile platform with two motors, and three sensors, one frontal, and one in each side. It uses ultrasound sensors and thus the perception of barrier or obstacle exists only within a scope zone of the sensor. Moreover the intensity of the sensor measure is inversely proportional to the distance of the object.

This simulation environment has served initially to knowledge acquisition through observation data input and output, and observation of robot behavior in several situations. Afterwards, two experiments were performed to validate the D-FCM and Fuzzy System navigation systems. In the first and second experiments, two different scenarios with static and dynamics obstacles have been simulated. The first experiment a dynamic obstacle is randomly inserted into the environment, during the robot navigation.

The results are presented in figures 3 and 4, they describe the path take by the fuzzy and D-FCM with hebbian learning algorithm. At Figure 3, the obstacle with coordinates $(7.25,96)$ is surprise, thus after the mobile executed half of the trajectory, this object enter in scenario. In Figure 4, these figures, the left graphic shows the scenario (x-y plan) with the initial about $(15,0)$ and end point near $(0,160)$ of the robot trajectory. The graphic shows the dynamic trajectory made by the robot. The apparent flaws in the trajectory represent the speedup, when sensors do not detect an obstacle and the robot accelerates. In all experiments, we consider that the robot successfully attains the target point if its final position is into a horizontal interval $[-8,+8]$ around the desired end point.

In the every scenario, example (figures 3,4 ), there is a critical situation with a surprise obstacle around the position $y=100$. In the figure 3 the robot must to take the decision of to move straight, pass between the two obstacles and immediately to turn left to avoid a frontal barrier and to attain the target point. By analyzing the results in figure $3 \mathrm{a}$ and $3 \mathrm{~b}$ we note that the robot takes the correct decisions.

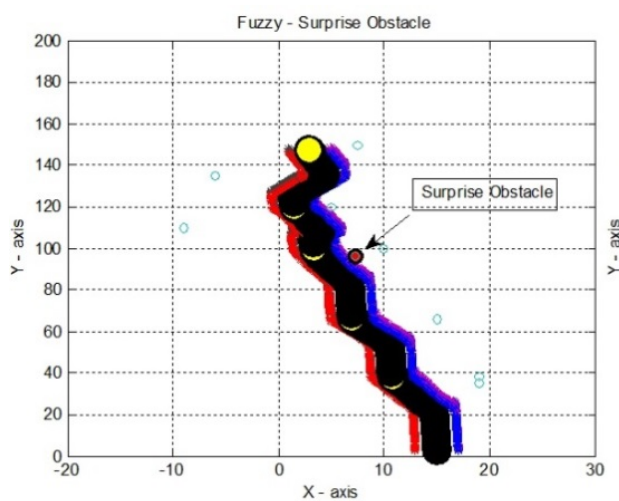

(a)

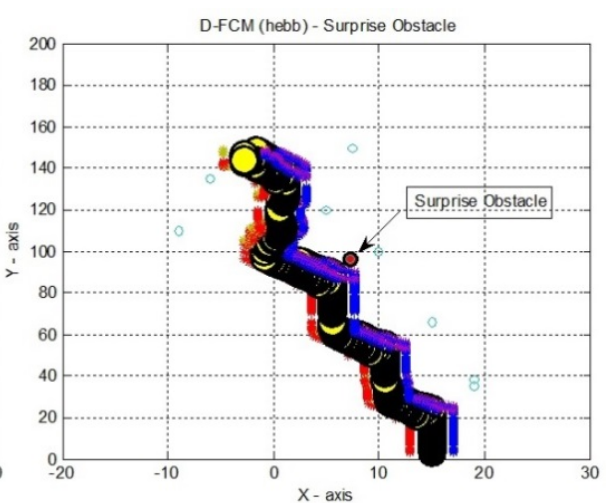

(b)

Fig. 3. a) Fuzzy Classic Architecture b) D-FCM Architecture with Hebbian Learning Algorithms 
In second case, one dynamic obstacle is in the scenario starting about in position $(10,83)$ and finishing about in position $(3,83)$, figure $4 \mathrm{a}$ and $4 \mathrm{~b}$ show the correct decisions in all maneuvers. In both cases, the robot motion trend is to move straight to the end point, but an. the robot takes the correct decision to turn in order to avoid a collision but it also maintains the motion trend of follow a straight line.

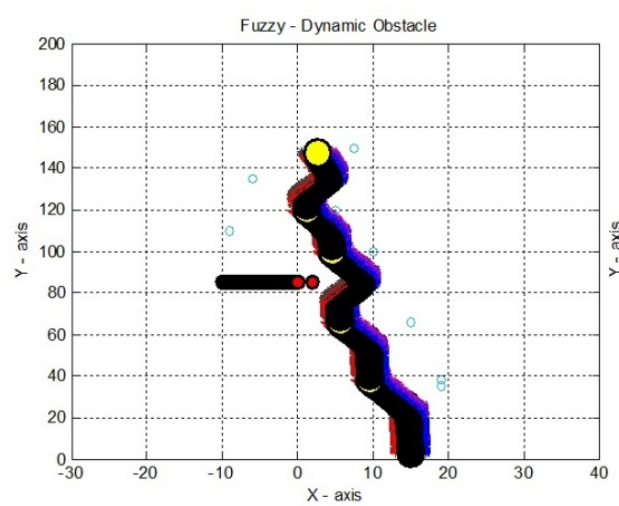

(a)

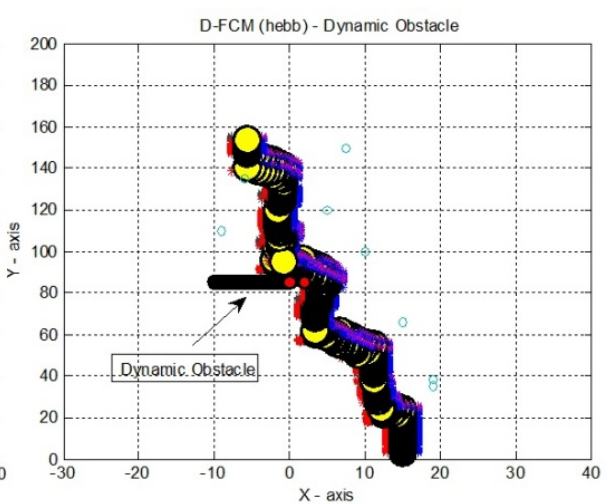

(b)

Fig. 4. a) Fuzzy Classic Architecture; b) D-FCM Architecture with Hebbian Learning Algorithms

\section{Conclusion}

This paper developed an autonomous navigation system based on a new type of fuzzy cognitive maps, named dynamic fuzzy cognitive map, D-FCM. The developed DFCM approach adds new types of relationships and concepts into a classical FCM that allows modeling the human ability of to take decision in presence of random events. The human knowledge is represented by a rule based system that is triggered when critical situation occurs. As a result, the inference engine adds temporally concepts and relationships into the FCM. This approach is a contribution of this paper to the intelligent control area. It is not restricted to navigation systems and can be applied to model intelligent system with online decision making. In accordance with the results presented in this paper, we can conclude that the proposed D-FCM architecture constitutes a flexible and robust tool to navigation system able for navigate in vagueness and uncertainty in environment using dynamic planning. One of the main advantages of the proposed approach is that the knowledge acquisition and representation is simplified by the use of FCM models. Moreover the resulting fuzzy cognitive maps are also easy to implement and run. Thus, it is easily embedded in a hardware robot.

Future works addresses more functions and goals added in the robot, example energy management and reach targets. New scenarios, with more complexity, adding borders and more robots (agents). Finally, due to easy implement real scenarios can be development. 


\section{References}

1. Passino, M.K., Yourkovich, S.: Fuzzy control. Addison-Wesley, Menlo Park (1997)

2. Russell, S.J., Norvig, P.: Artificial intelligence: a modern approach. Prentice Hall, Englewood Cliffs (1995)

3. Calvo, R.: Arquitetura híbrida inteligente para navegação autônomo de robôs. Dissertação (Mestrado em Ciências de Computação e Matemática Computacional). IMC-USP (2007)

4. Asami, S.: Robots in Japan: Present and Future. IEEE Robotics and Automation Magazine 1(2), 22-26 (1994)

5. Schraff, R.D.: Mechatronics and Robotics for Service Applications. IEEE Robotics and Automation Magazine 1(4), 31-35 (1994)

6. Mandow, A., Gomes-de-Gabriel, J.M., Martinéz, J.L., Muñoz, V.F., Ollero, A., García-Cerezo, A.: The Autonomous Mobile Robot AURORA for Greenhouse Operation. IEEE Robotics and Automation Magazine 3(4), 18-28 (1996)

7. Mendonça, M., Angélico, B.A., Arruda, L.V.R., Neves Jr., F.: A Subsumption Architecture to Develop Dynamic Cognitive Network-Based Models With Autonomous Navigation Application. Journal of Control, Automation and Electrical Systems 1, 3-14 (2013)

8. Kosko, B.: Fuzzy Cognitive Maps. Int. J. Man-Machine Studies 24, 65-75 (1986)

9. Siraj, A., Bridges, S., Vaughn, R.: Fuzzy Cognitive Maps for Decision Support in an Intelligent Intrusion Detection System. Technical Report, Department of Computer Science, Mississippi State University. MS 39762 (2001)

10. Malhotra, R., Sarkar, A.: Development of a fuzzy logic based mobile robot for dynamic obstacle avoidance and goal acquisition in an unstructured environment. In: Proceedings of the 2005 IEEE/ASME International Monterey, California, USA, pp. 24-28 (July 2005)

11. Astudillo, L., Castillo, O., Melin, P., Alanis, A., Soria, J., Aguilar, L.T.: Intelligent Control of an Autonomous Mobile Robot using Type-2 Fuzzy Logic. Engineering Letters 13(2) (2006)

12. Min, H.Q., Hui, J.X., Lu, Y.-S., Jiang, J.: Probability Fuzzy Cognitive Map for Decisionmaking in Soccer Robotics. In: Proceedings of the IEEE/WIC/ACM International Conference on Intelligent Agent Technology, IAT 2006 (2006), 0-7695-2748-5/06

13. Pipe, A.G.: An Architecture for Building "Potential Field" Cognitive Maps in Mobile Robot Navigation. Adaptive Behavior 8(2), 173-203 (2000)

14. Yeap, W.K., Wong, C.K., Schmidt, J.: Initial experiments with a mobile robot on cognitive mapping. In: Proceedings of the 2006 International Symposium on Practical Cognitive Agents and Robots, Perth, Australia, November 27-28 (2006)

15. Mendonça, M., Arruda, L.V.R., Neves, F.A.: Autonomous Navigation System Using Event Driven-Fuzzy Cognitive Maps. Applied Intelligence (Boston) 37, 175-188 (2011)

16. Axelrod, R.: Structure of Decision: the Cognitive Maps of Political Elites. Princeton University Press, New Jersey (1976)

17. Chun-Mei, L.: Using fuzzy cognitive map for system control. In: WTOS 7, vol. 12, pp. 1504-1515 (December 2008)

18. Papageorgiou, E.: Learning Algorithms for Fuzzy Cognitive Maps. IEEE Transactions on Systems and Cybernetics 42, 150-163 (2012)

19. Harisha, S.K., Ramkanth Kumar, P., Krishna, M., Sharma, S.C.: Fuzzy Logic Reasoning to Control Mobile Robot on Pre-defined Strip Path. In: Proceedings of World Academy of Science, Engineering and Technology 32 (August 2008) ISSN 2070-3740 